\title{
Estoque de duas espécies arbóreas ameaçadas de extinção aos 30 anos após a exploração na Amazônia oriental
}

\author{
Stock of two timber species threatened by extinction \\ 30 years after logging in the eastern Amazon Region
}

\author{
Jaqueline Macedo Gomes', João Olegário Pereira de Carvalho² ${ }^{2}$ Ademir Roberto Ruschel ${ }^{3}$, \\ José Natalino Macedo Silva ${ }^{2}$, Edson Marcos Leal Soares Ramos ${ }^{4}$, Tatiana Cunha Castro ${ }^{5}$, \\ Ian Samuel Thompson ${ }^{6}$ e Lucas José Mazzei de Freitas ${ }^{3}$
}

\section{Resumo}

O manejo sustentável das espécies ameaçadas de extinção deve ser utilizado como uma estratégia de conservação, portanto é de fundamental importância conhecer seus comportamentos em relação à exploração florestal e à taxa de recuperação do seu estoque dentro do ciclo de corte estabelecido para a floresta. Assim, avaliou-se a abundância, área basal, volume e a distribuição diamétrica de Hymenaea parvifolia Huber e Hymenolobium excelsum Ducke, consideradas ameaçadas de extinção e que tiveram suas madeiras colhidas em duas áreas experimentais da Embrapa Amazônia Oriental, localizadas na Floresta Nacional do Tapajós, sendo uma no Km 67 e outra no Km 114 da BR 163, ambas no município de Belterra, Pará. Foram utilizados dados de inventários realizados a $100 \%$ de intensidade no $\mathrm{km} 114$ (em 1981, um ano antes da exploração, e em 2014, 32 anos após a exploração) e no km 67 (em 1975, quatro anos antes da exploração, e em 2009, 30 anos após a exploração). Comparou-se a abundância, área basal, distribuição diamétrica dos indivíduos e do volume entre os dois inventários em cada área. O período de 30 anos após a exploração florestal não foi suficiente para Hymenaea parvifolia recuperar o número de árvores, a área basal e o volume existente antes da exploração, nas duas áreas experimentais avaliadas, considerando a alta intensidade de colheita de madeira. Atualmente a espécie tem volume disponível para colheita, na área do $\mathrm{Km} 67$, porém não possui estoque em crescimento que possa assegurar novas colheitas em futuro próximo, necessitando de atenção especial no manejo de sua população. Na área do $\mathrm{Km} \mathrm{114,} \mathrm{a} \mathrm{espécie} \mathrm{possui} \mathrm{um} \mathrm{estoque} \mathrm{que} \mathrm{garante} \mathrm{a} \mathrm{colheita} \mathrm{no} \mathrm{segundo} \mathrm{corte,} \mathrm{porém} \mathrm{recomenda-se} \mathrm{que}$ sua exploração não ocorra antes de recuperar completamente a estrutura de sua população original. A alta intensidade de colheita não permitiu que Hymenolobium excelsum recuperasse o número de árvores existente antes da exploração nas duas áreas experimentais, no período de 30 anos. A espécie possui estoque em crescimento que garante a sua exploração nos próximos cortes, seguindo a legislação em vigor, porém sugere-se que seja colhida após recuperar a estrutura de sua população original.

Palavras-chave: Estrutura diamétrica, Volume de árvores, ciclo de corte, Hymenaea parvifolia, Hymenolobium excelsum, Manejo Florestal.

\footnotetext{
Abstract

Sustainable management of species threatened with extinction should be used as a conservation strategy. It is essential to know the behavior of these species in relation to forest logging and the recovery rate of its stock over the cutting cycle established for the forest. Thus abundance, basal area, volume and diameter distribution of Hymenaea parvifolia Huber and Hymenolobium excelsum Ducke, considered species threatened with extinction and which were harvested for timber at Embrapa Amazônia Oriental's experimental sites at Km

1Pesquisadora Doutora. SEMAS/PA - Secretaria do Estado de Meio Ambiente e Sustentabilidade. Travessa Lomas Valentinas, 2717 - 66.093-677 - Belém, PR, Brasil. E-mail: jaquelinemacedogomes@hotmail.com.

2Professor do Programa de Pós-Graduação em Ciências Florestais. UFRA - Universidade Federal Rural da Amazônia. Av. Presidente Tancredo Neves, 2501 - 66.077-530 - Belém, PR, Brasil. E-mail: olegario@pq.cnpq.br; silvanatalino734@gmail.com.

${ }^{3}$ Pesquisador Doutor. EMBRAPA Amazônia Oriental - Empresa Brasileira de Pesquisa Agropecuária. Trav. Dr. Enéas Pinheiro s/n - Caixa Postal: 48 - 66.095-100 - Belém, PA, Brasil. E-mail: ademir.ruschel@embrapa.br; lucas.mazzei@embrapa.br.

${ }^{4}$ Professor Associado do Departamento de Ciências Exatas e Naturais. UFRA - Universidade Federal Rural da Amazônia. Av. Presidente Tancredo Neves, 2501 - 66.077-530 - Belém, PR, Brasil. E-mail: ramosedson@gmail.com.

${ }^{5}$ Doutoranda em Ciências Florestais. UFRA - Universidade Federal Rural da Amazônia. Av. Presidente Tancredo Neves, 2501 - 66.077-530 - Belém, PR, Brasil. E-mail: ccastro.tatiana@gmail.com.

${ }^{6}$ Pesquisador Mestre em Ciências Florestais. TNC - The Nature Conservancy. Av. Nazaré, 280 - 66.035-170 - Belém, PA, Brasil. E-mail: ithompson@tnc.org.
} 
Gomes et al. - Estoque de duas espécies arbóreas ameaçadas de

extinção aos 30 anos após a exploração na Amazônia oriental

114 and Km 67 of BR 163 in the Tapajos National Forest, municipality of Belterra, Pará were studied. Data from 100\% inventories from Km 114 (from 1981- one year before logging and in 2014- 32 years after logging) and from Km 67 (in 1975 - four years before logging and in 2009- 30 years after logging) were evaluated. Diameter distribution of abundance, basal area and volume were compared between the two inventories of each site. The period of 30 years after logging was not sufficient for Hymenaea parvifolia to recover prelogging tree numbers, basal area or volume in both sites, due to the high logging intensity applied. The species has a volume available for harvesting at $\mathrm{Km} 67$; however it does not have a growing stock that could ensure new harvests in the near future, thus needing special management attention of the population. At Km 114, the species showed a stock to support the second harvest, but we recommend its harvest after recovering its pre-logging population structure. The high logging intensity meant that Hymenolobium excelsum did not recover pre-logging tree numbers at both sites in the 30 years. However the species showed growing stock that could guarantee its future logging, following current legislation, but we also recommend its harvest after recovering its pre-logging population structure.

Keywords: Diametric structure, tree volume, cutting cycle, Hymenaea parvifolia, Hymenolobium excelsum, Forest management.

\section{INTRODUÇÃO}

A prática de manejo florestal sustentável concilia a utilização econômica dos recursos naturais com a conservação dos ecossistemas, em benefício da sociedade. Assim, o silvicultor deve considerar os efeitos que o sistema silvicultural terá sobre a biodiversidade, uma vez que os sistemas são fundamentados na remoção seletiva de certas espécies. Populações viáveis de todas as espécies devem ser mantidas, procurando sempre garantir a conservação da biodiversidade (HIGMAN et al. 2015).

Os benefícios das florestas manejadas de forma sustentável são reconhecidos pela sociedade, governos e mercados. O manejo florestal é incorporado em estratégias de conservação que promovem a proteção da biodiversidade, mitigação das alterações climáticas, o abastecimento de madeira e geração de renda no meio rural (PUTZ et al., 2012).

Apesar dos benefícios citados, Braz et al. (2012a) afirmam que os planos de manejo, como atualmente preconizados, não são sustentáveis, pois não consideram o crescimento real das espécies individualmente. Segundo Braz et al. (2012b), os volumes determinados para extração são arbitrários, e o conceito de manejo de florestas não é assegurado nos planos de manejo. Para garantir a sustentabilidade dos planos é indispensável existir previsão do percentual de volume extraído por espécie que poderá ser recuperado no período de tempo definido pelo ciclo de corte.

A recuperação da floresta, assim como a recuperação das populações das espécies exploradas, no ciclo de corte considerado, não deve ser avaliada somente pelas informações do incremento. É necessário também considerar cuidadosamente a distribuição diamétrica das árvores remanescentes (BRAZ et al., 2012b). Lima e Leão (2013) consideram que a análise do comportamento dos diferentes táxons em cada classe diamétrica subsidia a escolha do sistema silvicultural, incluindo a intensidade de exploração, e os métodos silviculturais a serem adotados, de maneira que a floresta seja manejada corretamente. Os autores acrescentam ainda que o conhecimento da estrutura e das taxas de crescimento é a base para o seu manejo.

Com a finalidade de auxiliar a conservação das espécies consideradas ameaçadas, foram estabelecidas pelo Ministério do Meio Ambiente normas específicas para o seu manejo. A portaria 443, de 17 de dezembro de 2014 (BRASIL, 2014), reconhece e apresenta a "Lista Nacional Oficial de espécies da flora brasileira Ameaçadas de Extinção" e estabelece a proibição de coleta, corte, transporte, manejo, beneficiamento e comercialização de espécies classificadas nas categorias Criticamente em Perigo (CR), Em Perigo (EM) e Vulneráveis (VU). A Instrução Normativa $\mathrm{n}^{\circ} 1$ de fevereiro de 2015 (BRASIL, 2015) estabelece limite de colheita para as espécies classificadas na categoria Vulnerável-VU.

O manejo das populações ameaçadas deve ser utilizado como uma estratégia de conservação, assim, é fundamental conhecer o comportamento das espécies em relação à exploração e à taxa de recuperação do seu estoque, dentro do ciclo de corte estabelecido para a floresta. Com o monitoramento da dinâmica das espécies exploradas, é possível constatar o seu status e assim tomar decisões de conservação, impedindo sua extinção na área e garantindo o manejo de sua população. A adoção de tratamentos silviculturais pode ser benéfica, estimulando o crescimento e a regeneração por meio da abertura do dossel. 
Na presente pesquisa avaliou-se o estoque das populações das espécies Hymenaea parvifolia Huber e Hymenolobium excelsum Ducke, ambas classificadas na categoria de Espécie Vulnerável na lista oficial de espécies ameaçadas de extinção, e que tiveram indivíduos colhidos em duas áreas de manejo na Floresta Nacional do Tapajós. A avaliação foi feita em duas ocasiões, uma antes da exploração florestal e outra aos 30 anos após a exploração para, de acordo com as alterações ocorridas nesse período, informar sobre a possibilidade de continuar extraindo a madeira dessas espécies, sem correr o risco de extinção na área, ou confirmar a permanência das mesmas como ameaçadas de extinção.

\section{MATERIAL E MÉTODOS}

\section{Áreas de estudo}

A pesquisa foi realizada em duas áreas experimentais da Embrapa Amazônia Oriental, na Floresta Nacional do Tapajós, município de Belterra, Pará, sendo uma localizada à altura do Km 67 da BR 163 - Rodovia Santarém-Cuiabá e a outra localizada à altura do Km 114 da mesma rodovia.

\section{Floresta Nacional do Tapajós - Área Experimental Km 114}

A área experimental do km 114 possui 180 ha e está localizada entre as coordenadas geográficas $03^{\circ} 18^{\prime} 32^{\prime \prime}$ e $03^{\circ} 19^{\prime} 21^{\prime \prime}$ de latitude sul e $54^{\circ} 56^{\prime} 28^{\prime \prime}$ e $54^{\circ} 56^{\prime} 15^{\prime \prime}$ de longitude a oeste. Os solos que predominam a região de Belterra são do tipo Latossolo amarelo distrófico e Argissolo amarelo distrófico (OLIVEIRA JUNIOR; CORREA, 2001). O clima da região do Tapajós é do tipo Am, segundo a classificação de Köppen-Geiger (KOTTEK et al., 2006), com temperatura média anual de 27 ${ }^{\circ} \mathrm{C}$, umidade relativa do ar de $87 \%$ e precipitação pluviométrica média anual de $1.982 \mathrm{~mm}$ (INMET, 2017). A vegetação da área de estudo é do tipo Floresta Ombrófila Densa (IBGE, 2012).

\section{Floresta Nacional do Tapajós - Área Experimental Km 67}

A área experimental do Km 67 possui 91 ha e está localiza-se na Floresta Nacional do Tapajós, também no município de Belterra, Pará, à altura do Km 67 da BR 163, Rodovia Santarém-Cuiabá, entre as coordenadas geográficas $02^{\circ} 53^{\prime} 03,09^{\prime \prime}$ de latitude sul e $54^{\circ} 55^{\prime} 30,10^{\prime \prime}$ de longitude oeste. As características de solo, clima e vegetação são as mesmas da Área Km 114.

\section{Espécies estudadas}

Hymenaea parvifolia Huber e Hymenolobium excelsum Ducke foram selecionadas para o estudo por terem sido exploradas nas áreas experimentais e por estarem contidas na lista vermelha da União Internacional de Conservação da Natureza (IUCN, 2012) e na lista de espécies ameaçadas de extinção do Ministério do Meio Ambiente (BRASIL, 2014), além disso, foram colhidas nas duas áreas experimentais. H. parvifolia e H. excelsum foram exploradas em 1979 na área experimental do Km 67 e em 1982 na área do Km 114. Nas áreas de estudo a identificação botânica foi feita por parabotânicos qualificados da Embrapa Amazônia Oriental.

H. parvifolia pertence à família Fabaceae, conhecida vulgarmente como jutaí-mirim, possui ampla distribuição geográfica, ocorrendo nos estados do Acre, Amazonas, Pará, Rondônia, Roraima, Maranhão, Piauí e Mato Grosso (FERNANDES et al., 2015). É uma espécie de grande porte, suas árvores atingem DAP entre 70-100 cm e altura entre 25 e 40 metros de altura (SALOMÃO, et al.; 1995). A floração ocorre no período de agosto a fevereiro, a frutificação durante o ano todo e a disseminação de agosto a março (LEÃO; CARVALHO, 2001). A dispersão é barocórica e zoocórica (SALOMÃO et al., 2007) e a temperatura ótima para germinação da espécie está entre 25 e $35^{\circ} \mathrm{C}$ (SILVA; CESARINO, 2016). A espécie pertence ao grupo ecológico das intermediárias que, segundo Jardim et al. (1996), possuem características que as posicionam entre aquelas que necessitam de muita radiação solar para se desenvolver e aquelas que podem se desenvolver sob sombra e, segundo Silva et al. (2007), possuem também melhores chances de regeneração sob dossel fechado. Possui madeira de alto valor comercial, utilizada em movelaria de luxo, confecção de instrumentos diversos e nas indústrias aeronáutica e naval, além de possuir utilização não madeireira, servindo de alimento para o homem e para a fauna (SALOMÃO, et al.; 1995).

H. excelsum pertence à família Fabaceae, conhecida vulgarmente como angelim-da-mata e fava-folha-fina. Ocorre nos estados do Amapá, Amazonas e Pará (FERREIRA et al., 2004a), sendo utili- 
zada na construção em geral, tornearia, marcenaria, carpintaria, dormentes, tacos e objetos de adornos (FERREIRA et al., 2004b). A floração tem início no período de março a junho com a frutificação ocorrendo de maio a outubro e a disseminação de dezembro a fevereiro (LEÃO et al., 2015; VIEIRA et al., 2002). A espécie pertence ao grupo das tolerantes à sombra (LOPES et al., 2001).

\section{Atividades realizadas na área experimental Km 114}

$\mathrm{Na}$ área do Km 114 o experimento de manejo florestal foi instalado em 144 ha no ano de 1981. Nesse mesmo ano foi realizado inventário a $100 \%$ de intensidade considerando as árvores com DAP (diâmetro a 1,30 m do solo) $\geq 45 \mathrm{~cm}$; foi feito também corte de cipós em toda a área. Em 1982 foi realizada uma exploração florestal planejada, com a colheita de 38 espécies e média de 12,5 árvores por ha, com volume de árvores em pé de $90 \mathrm{~m}^{3} \mathrm{ha}^{-1}$ (CARVALHO, 1987). Esse volume foi muito superior ao permitido atualmente pela legislação brasileira, que é de, no máximo, $30 \mathrm{~m}^{3} \mathrm{ha}^{-1}$ (BRASIL, 2006). Na época da exploração (1982) a legislação determinava apenas o diâmetro mínimo de corte $(45 \mathrm{~cm})$, que se aplicava a qualquer espécie com madeira de valor comercial, mas não havia limite de volume. Em 1993 e 1994 foram realizados desbastes seletivos-sistemáticos na área (COSTA et al., 2001). Em 2014 foi realizado o segundo inventário a 100\% de intensidade. Neste trabalho foram utilizados dados dos inventários realizados em 1981, antes da exploração, e em 2014, 32 anos depois da exploração. As informações do inventário de 1981 foram obtidas da publicação de Silva et al. (1985).

\section{Atividades realizadas na área experimental $\mathrm{Km} 67$}

$\mathrm{Na}$ área Km 67, as pesquisas foram iniciadas em 1975, com a realização de inventário a $100 \%$ de intensidade para as árvores com DAP $\geq 45 \mathrm{~cm}$ e inventário diagnóstico da regeneração natural, utilizando um método malaio de amostragem linear em 64 ha (CARVALHO, 1980; CARVALHO et al., 1984). Em 1979 foi realizada a exploração florestal planejada nestes mesmos 64 ha, considerando duas intensidades de colheita: corte de todas as árvores com DAP $\geq 45 \mathrm{~cm}$ de 64 espécies com estoque comercial em 39 ha; e corte de todas as árvores com DAP $\geq 55 \mathrm{~cm}$ das mesmas 64 espécies nos 25 ha restantes (COSTA FILHO et al., 1980). A madeira colhida nos 64 ha gerou, em média, 72,5 $\mathrm{m}^{3} \mathrm{ha}^{-1}$ e uma intensidade de corte de 20,6 árvores por ha (COSTA FILHO et al., 1980). Em 2009 foi realizado o segundo inventário a 100\% de intensidade nos 64 ha.

Na presente pesquisa foram utilizados os dados de inventários realizados a $100 \%$ de intensidade em duas ocasiões: em 1975, antes da exploração, e em 2009, 34 anos de pesquisa.

\section{Análise dos dados}

A avaliação da estrutura da população das espécies ameaçadas foi feita por meio da análise da área basal $\left(\mathrm{m}^{2} \mathrm{ha}^{-1}\right)$, do volume $\left(\mathrm{m}^{3} \mathrm{ha}^{-1}\right)$, do número de indivíduos por hectare $\left(\mathrm{N} \mathrm{ha}^{-1}\right)$ e da distribuição diamétrica das árvores $\mathrm{DAP} \geq 45 \mathrm{~cm}$. Foi considerado intervalo de $10 \mathrm{~cm}$ entre as classes. As mesmas classes diamétricas foram utilizadas para analisar a distribuição do volume. No Km 114 o volume foi calculado utilizando a equação: $V=\mathrm{G} \times \mathrm{H} \times \mathrm{F}$, onde: $V$ é o volume $\mathrm{em} \mathrm{m}^{3} \mathrm{ha}^{-1}$; $\mathrm{G}$ é a área basal; H é a altura comercial do fuste e F é o fator de forma, que na presente pesquisa considerou-se igual a 0,7 (HEINSDIJK; BASTOS,1963). Devido à falta de informações sobre o fator de forma de cada uma das espécies, utilizou-se o fator de forma geral, representado pela constante 0,7 . A área basal (G) em 1981 foi estimada com dados das parcelas permanentes, pois não foi possível recuperar as informações do inventário $100 \%$. Na área experimental do Km 67, o volume foi calculado utilizando a equação de volume desenvolvida para a área: $\operatorname{lnV}=-7,81370+2,20385 \times \operatorname{lnd}$, onde: "V" é o volume, "d" é o DAP e "ln" é o logaritmo natural de base "e" (SILVA et al., 1984).

A análise estatística foi feita utilizando o teste t de Student para amostras pareadas, a 5\% de probabilidade, relacionando o ano antes da exploração com o ano após a exploração para os parâmetros abundância, área basal e volume. Para utilização do teste $\mathrm{t}$ foi realizado o teste de normalidade de Kolmogorov-Smirnov a 5\% de probabilidade e observada a existência de heterocedasticidade dos dados. Nos casos de comprovada a não normalidade dos dados e a heterocedasticidade, foi utilizado o teste não paramétrico de Mann-Whitney a 5\% de probabilidade.

As comparações entre as distribuições diamétricas do número de árvores e de volume nos dois inventários realizados a $100 \%$ de intensidade, em cada área e para cada espécie, foram realizadas por meio do teste de Kolmogorov-Smirnov, com nível de significância de 0,05. 


\section{RESULTADOS E DISCUSSÃO}

\section{Hymenaea parvifolia Huber (jutaí-mirim)}

Aos 32 anos após a exploração, na área experimental do Km 114, Hymenaea parvifolia não conseguiu recuperar a abundância, área basal e volume originais (Tabela 1), pois o crescimento dos indivíduos remanescentes foi insuficiente para permitir a recuperação dentro do período estabelecido como ciclo de corte da floresta, que de acordo com a Legislação Brasileira é de 35 anos (BRASIL, 2006).

A legislação atual permite a exploração das espécies ameaçadas classificadas na categoria "Vulnerável", no entanto, exige a manutenção de, pelo menos, 15\% do número de árvores por espécie na área efetiva de exploração da Unidade de Produção Anual, respeitando o limite mínimo de manutenção de 4 árvores por espécie a cada 100 ha (BRASIL, 2015). Embora a recuperação de $H$. parvifolia em volume seja de apenas 23\%, em número de indivíduos (N) ela chega a 52\% (Tabela 1), correspondendo a 17 árvores em cada 100 ha. Se na legislação o limite mínimo para manutenção é de 4 árvores em 100 ha, então na área de estudo, a espécie tem baixo risco de ser extinta, pois está recuperando seu estoque de forma gradativa, além de não ser considerada uma espécie rara, de acordo com a IN 05 de 2006 (BRASIL, 2006), assim a mesma poderia ser explorada em um próximo corte. No entanto, seria prudente esperar maior recuperação do estoque para realizar uma nova exploração e, assim, não causar prejuízo ao estado de conservação da espécie.

No $\mathrm{Km} \mathrm{67,} \mathrm{H.} \mathrm{parvifolia} \mathrm{não} \mathrm{recuperou} \mathrm{a} \mathrm{abundância,} \mathrm{nem} \mathrm{a} \mathrm{área} \mathrm{basal} \mathrm{e} \mathrm{nem} \mathrm{o} \mathrm{volume,} \mathrm{no}$ entanto, chegou a números próximos aos encontrados antes da exploração (Tabela 1). Por exemplo, em relação ao volume falta $0,037 \mathrm{~m}^{3} \mathrm{ha}^{-1}$ para recuperar o estoque, ou seja, $26 \%$ do volume colhido na área, que foi $0,142 \mathrm{~m}^{3} \mathrm{ha}^{-1}$ (COSTA FILHO et al., 1980). Os autores observaram que na área do Km 67 foi colhido 0,031 indivíduo de H. parvifolia por hectare, reduzindo a abundância para 0,078 indivíduos por ha, permanecendo assim até 2009, pois não houve mortes e nem ingressos durante o período (Tabela 1).

Tabela 1. Número total de árvores $(\mathrm{N})$, abundância $\left(\mathrm{N} \mathrm{ha} \mathrm{a}^{-1}\right)$, área basal $\left(\mathrm{m}^{2} \mathrm{ha}^{-1}\right)$ e volume $\left(\mathrm{m}^{3}\right.$ ha $\left.{ }^{-1}\right)$ de $H y m e n a e a$ parvifolia Huber em inventários realizados a $100 \%$ de intensidade, dos indivíduos com DAP $\geq 45 \mathrm{~cm}$, nas áreas experimentais do Km 114 e do $\mathrm{Km} 67$ na Floresta Nacional do Tapajós.

Table 1. Total number of trees $(\mathrm{N})$, abundance $\left(\mathrm{N} \mathrm{ha}^{-1}\right)$, basal area $\left(\mathrm{m}^{2} \mathrm{ha}^{-1}\right)$ and volume ( $\left.\mathrm{m} 3 \mathrm{ha}-1\right)$ of Hymenaea parvifolia Huber from $100 \%$ inventories of individuals with $\mathrm{DBH} \geq 45 \mathrm{~cm}$, in the $\mathrm{Km} 114$ and $\mathrm{Km} 67$ experimental areas in the Tapajós National Forest.

\begin{tabular}{|c|c|c|c|c|c|}
\hline Áreas & Anos & Número total (N) & Abundância (N ha-1) & Área basal $\left(\mathrm{m}^{2} \mathrm{ha}^{-1}\right)$ & Volume $\left(m^{3} h a^{-1}\right)$ \\
\hline \multirow{4}{*}{$\mathrm{Km} 114$} & 1981 & 48 & 0,336 & $0,070^{*}$ & 2,227 \\
\hline & 2014 & 25 & 0,174 & 0,047 & 0,506 \\
\hline & P-valor & - & $0,155(2)$ & $0,582(2)$ & $0,010(1)$ \\
\hline & \% de recuperação & 52 & 52 & 67 & 23 \\
\hline \multirow{4}{*}{ Km 67} & 1975 & 7 & 0,109 & 0,042 & 0,514 \\
\hline & 2009 & 5 & 0,078 & 0,038 & 0,477 \\
\hline & P-valor & - & $0,524(2)$ & $0,862(2)$ & $0,889(2)$ \\
\hline & \% de recuperação & 71 & 72 & 91 & 93 \\
\hline
\end{tabular}

* área basal estimada com dados obtidos em parcelas permanentes; (I) Teste $\mathrm{t}$ - significativa a 5\%; (2) Teste $\mathrm{t}$ - não significativo 5\%

A estrutura diamétrica da espécie no Km 114 foi modificada pela exploração e aos 32 anos após a colheita da madeira a distribuição não voltou a se assemelhar àquela que existia antes da exploração (Figura 1). Estatisticamente, existe diferença significativa entre as distribuições diamétricas das árvores nos inventários de 1981 (antes da exploração) e 2014 (32 anos após a exploração), considerando um nível de significância de 5\%. Esse resultado está de acordo com a afirmativa de Orellana et al. (2014), de que as características ecológicas das espécies influenciam na distribuição diamétrica, e que as técnicas de manejo aplicadas à população podem descaracteriza-la. Embora até os 32 anos após a exploração a espécie não tenha atingido uma estrutura diamétrica semelhante à original, o seu estoque de madeira atual poderá compor a próxima colheita. Porém, sugere-se que a espécie não seja colhida no segundo corte na área. Cortes futuros precisam ser estimados.

No Km 67, a distribuição diamétrica das árvores de H. parvifolia foi modificada com a exploração e não recuperou sua forma inicial, pois ainda faltam indivíduos nas duas primeiras classes de diâmetro (Figura 1). Entretanto, não existe diferença significativa entre as abundâncias de 1975 e de 2009, considerando um nível de significância de 5\%. 


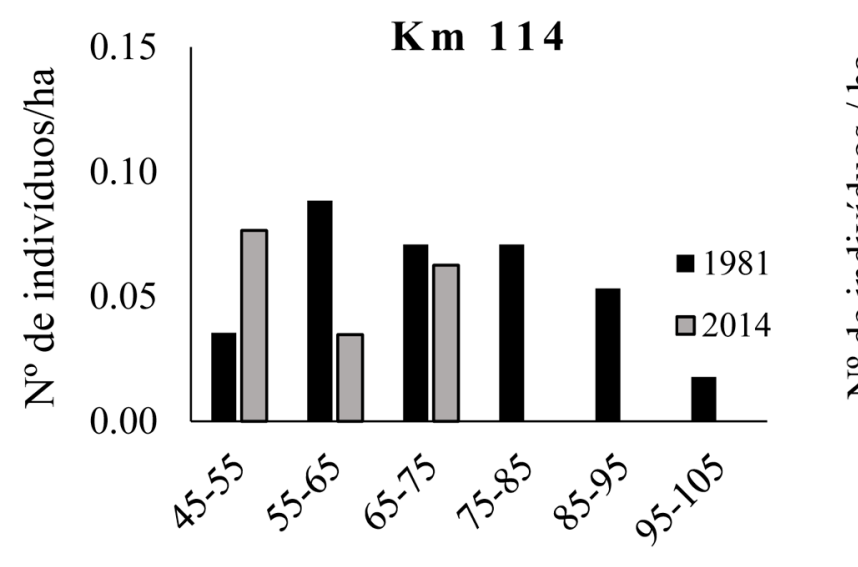

Classes de diâmetro $(\mathrm{cm})$

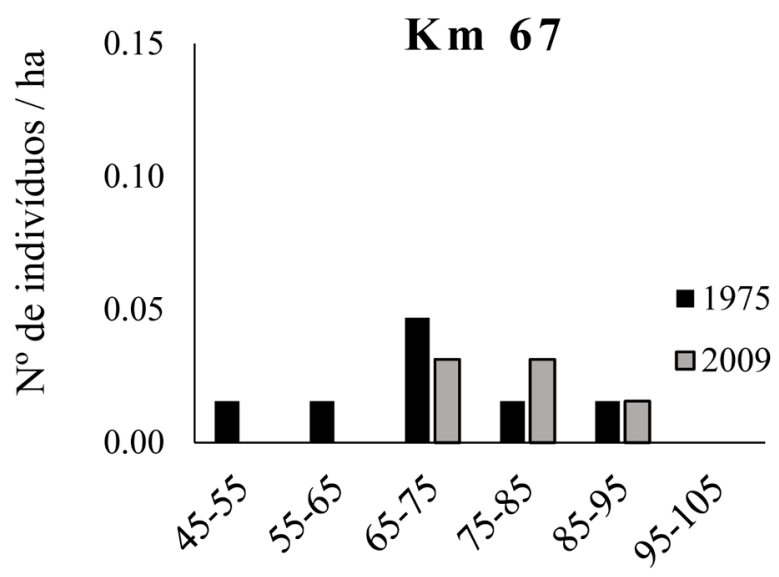

Classes de diâmetro (cm)

Figura 1. Distribuição diamétrica do número de árvores de Hymenaea parvifolia Huber nos inventários realizados a 100\% de intensidade na área experimental do km 114 e do Km 67 na Floresta Nacional do Tapajós.

Figure 1. Diameter distribution of tree number of Hymenaea parvifolia Huber from $100 \%$ intensity inventories in the km 114 and Km 67 experimental areas in the Tapajós National Forest.

Os tratamentos silviculturais diminuem a competição e estimulam o crescimento e a regeneração das espécies comerciais (PARIONA et al., 2003; SCHWARTZ et al. 2012), assim, a aplicação de tratamentos na área de estudo poderia ser realizada para testar a resposta da vegetação, considerando que para a exploração florestal a resposta não foi significativa.

Há uma significativa diferença entre os estoques de volume da área do Km 114 entre o ano de 1981 (antes da exploração) e 2014 (32 anos após a exploração), a um nível de significância de 5\% (Figura 2). Mas, apesar de a espécie não ter recuperado o seu volume original, é de se esperar que em longo prazo isso ocorra, pois observa-se que na classe de diâmetro 45-55 cm o número de árvores registrado em 2014 foi maior que em 1981, e na classe $65-75 \mathrm{~cm}$ falta 0,137 $\mathrm{m}^{3} \mathrm{ha}^{-1}$ para atingir o volume de 1981, antes da exploração. Baixas taxas de recuperação do volume colhido, semelhantes às registradas na presente pesquisa, foram observadas por Brienen e Zuidema (2007), estudando projeções de crescimento de três espécies arbóreas na Amazônia boliviana. Dauber et al. (2005), também destacaram baixa taxa de recuperação do volume das espécies exploradas, também na Bolivia, onde em 20 anos a recuperação dos estoques foi de apenas 4 a $28 \%$ do volume original.
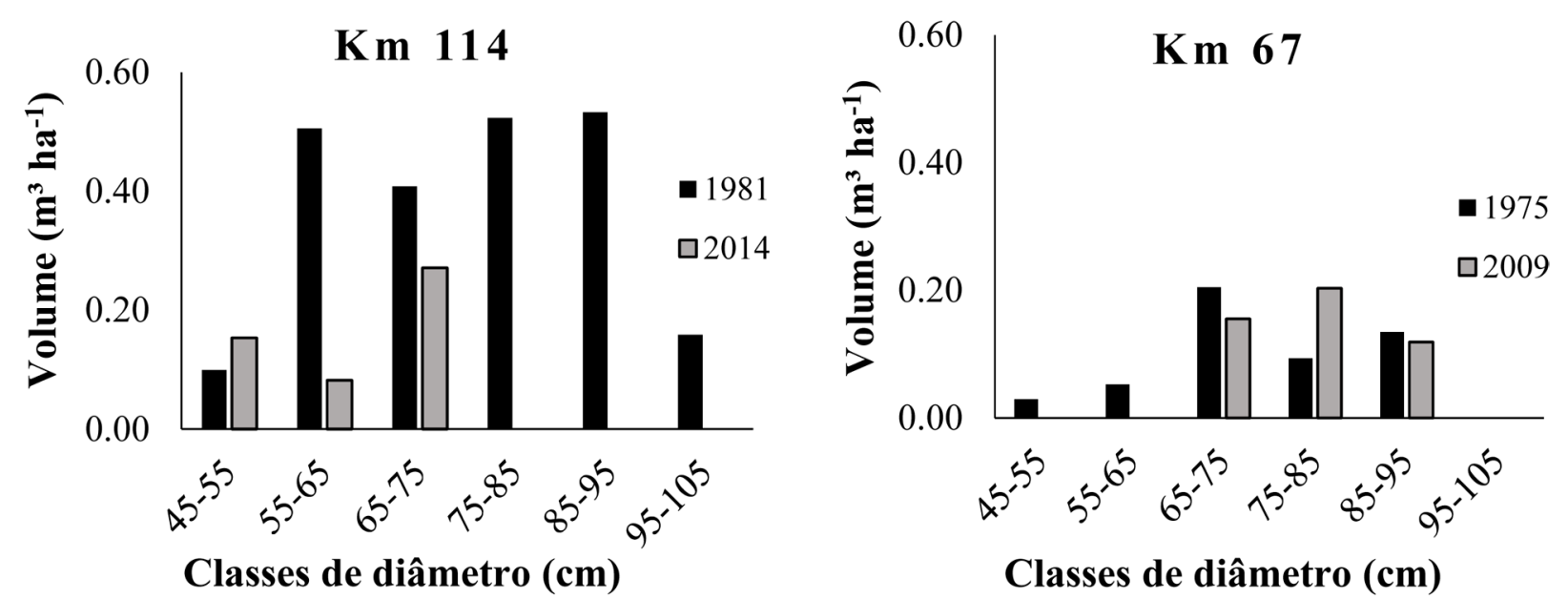

Figura 2. Volume de Hymenaea parvifolia Huber obtidos nos inventários a $100 \%$ de intensidade realizados na área experimental do Km 114 e Km 67 na Floresta Nacional do Tapajós.

Figure 2. Volume of Hymenaea parvifolia Huber from $100 \%$ intensity inventories in the $\mathrm{Km} 114$ and $\mathrm{Km} 67$ experimental areas in the Tapajós National Forest.

$\mathrm{Na}$ área do Km 67 não houve diferença significativa ao nível de 5\% entre as distribuições de volume dos inventários de 1975 e 2009. Não ocorreram indivíduos com diâmetro entre $45 \mathrm{~cm} \mathrm{e}$ $65 \mathrm{~cm}$ em 2009, porém o alto volume registrado nas classes maiores, principalmente na classe de 
75-85 cm nesse ano, superando o volume de 1975, possibilitou a semelhança entre os estoques em volume obtidos nos dois inventários (Figura 2).

A recuperação do volume de $H$. parvifolia ocorreu diferente nas duas áreas de pesquisa no período estudado. No km 114 ocorreram ingressos, porém a recuperação foi inferior à registrada no Km 67, onde a menor intensidade de exploração pode ter resultado em recuperação mais rápida.

\section{Hymenolobium excelsum Ducke (angelim-da-mata)}

$\mathrm{Na}$ área experimental do Km 114, H. excelsum não recuperou sua abundância, área basal e volume no período de 32 anos (1981-2014). A abundância e o volume são muito inferiores aos registrados no ano de 1981, antes da exploração (Tabela 2). Da mesma forma, no km 67, H. excelsum não recuperou sua abundância, área basal e volume em 34 anos (1975-2009). Esses parâmetros foram muito inferiores aos obtidos em 1975 (Tabela 2). Segundo Reis et al. (2010), o ciclo de corte de 30 a 35 anos é viável para o Km 67, desde que sejam colhidas madeiras de espécies diferentes daquelas colhidas na primeira exploração. Os autores realçam que a intensidade de exploração de 72,5 m ha ${ }^{-1}$ contribuiu para a não recuperação do estoque das espécies exploradas.

Tabela 2. Número total de árvores $(\mathrm{N})$, abundância $\left(\mathrm{N} \mathrm{ha}^{-1}\right)$, área basal $\left(\mathrm{m}^{2} \mathrm{ha}^{-1}\right)$ e volume $\left(\mathrm{m}^{3} \mathrm{ha}^{-1}\right)$ de Hymenolobium excelsum Ducke em inventários realizados a $100 \%$ de intensidade, dos indivíduos com DAP $\geq 45$ $\mathrm{cm}$, nas áreas experimentais do Km 114 e do Km 67 na Floresta Nacional do Tapajós.

Table 2. Total number of trees $(\mathrm{N})$, abundance $\left(\mathrm{N} \mathrm{ha}^{-1}\right)$, basal area $\left(\mathrm{m}^{2}\right.$ ha-1) and volume $\left(\mathrm{m}^{3}\right.$ ha-1 $)$ of Hymenolobium excelsum Ducke from $100 \%$ inventories of individuals with $\mathrm{DBH} \geq 45 \mathrm{~cm}$, in the $\mathrm{Km} 114$ and $\mathrm{Km} 67$ experimental areas in the Tapajós National Forest.

\begin{tabular}{|c|c|c|c|c|c|}
\hline Áreas & Anos & Número total (N) & Abundância (N ha-1) & Área basal $\left(\mathrm{m}^{2} \mathrm{ha}^{-1}\right)$ & Volume $\left(\mathrm{m}^{3} \mathrm{ha}^{-1}\right)$ \\
\hline \multirow{4}{*}{$\mathrm{Km} 114$} & 1981 & 56 & 0,389 & $0,047^{*}$ & 2,567 \\
\hline & 2014 & 12 & 0,083 & 0,022 & 0,252 \\
\hline & P-valor & - & $0,007(1)$ & $0,343(2)$ & $\leq 0,001(3)$ \\
\hline & \% de recuperação & 21 & 21,3 & 46,8 & 9,8 \\
\hline \multirow{4}{*}{ Km 67} & 1975 & 4 & 0,063 & 0,037 & 0,475 \\
\hline & 2009 & 2 & 0,031 & 0,014 & 0,174 \\
\hline & P-valor & - & $0,418(4)$ & $0,282(4)$ & $0,280(4)$ \\
\hline & \% de recuperação & 50 & 49,2 & 37,8 & 36,6 \\
\hline
\end{tabular}

* Valor estimado com dados obtidos em parcelas permanentes; (I) Teste de Mann-Whitney - significativa a 5\%; (2) Teste de Mann-Whitney - não significativo 5\%; (3) Teste $\mathrm{t}$ - significativa a 5\%; (4) Teste $\mathrm{t}$ - não significativo $5 \%$.

Analisando a distribuição diamétrica, observa-se que houve uma grande redução do número de indivíduos em todas as classes (Figura 3), porém, apesar dessa redução, a espécie ainda possui um estoque em crescimento que poderá ser colhido no próximo corte e em outras futuras colheitas.

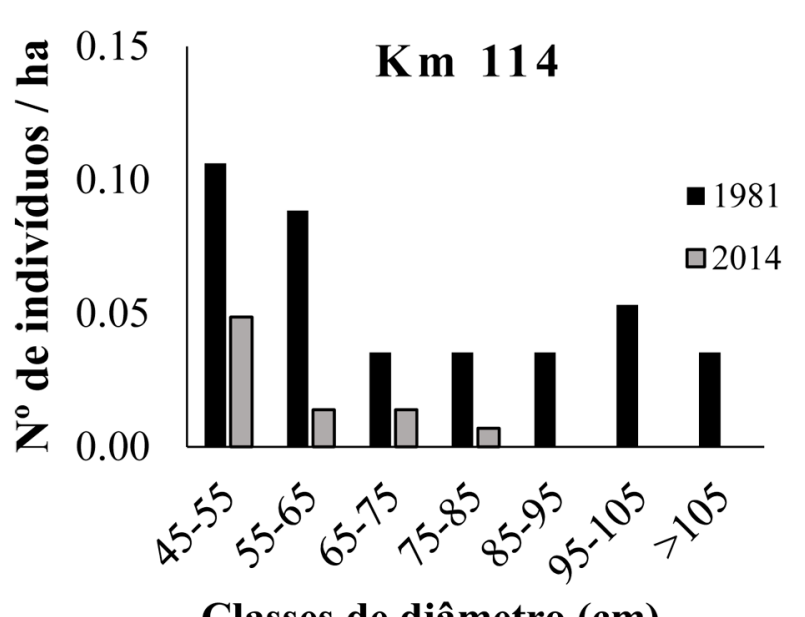

Classes de diâmetro $(\mathrm{cm})$

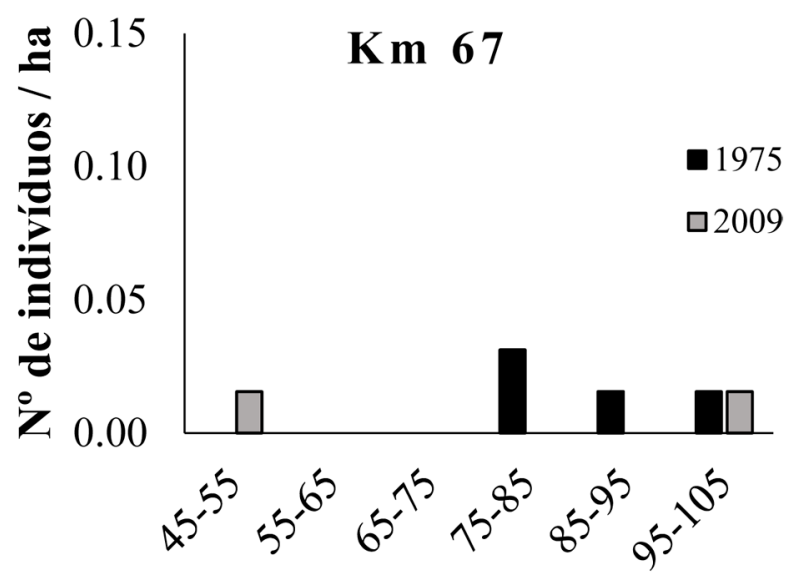

Classes de diâmetro (cm)

Figura 3. Distribuição diamétrica de árvores de Hymenolobium excelsum Ducke nos inventários a $100 \%$ de intensidade realizados na área experimental do Km 114 e Km 67 na Floresta Nacional do Tapajós.

Figure 3. Diameter distribution of tree number Hymenolobium excelsum Ducke from the $100 \%$ intensity inventories in the $\mathrm{Km} 114$ and Km 67 experimental areas in the Tapajós National Forest. 
No Km 67 a distribuição diamétrica de H. excelsum foi alterada pela exploração, e a sua população não recuperou a estrutura até 32 anos após a exploração. Antes da exploração, só havia indivíduos nas maiores classes de diâmetro (Figura 3). A falta de indivíduos com diâmetros inferiores a $75 \mathrm{~cm}$ em 1975 poderia ser uma justificativa para a espécie não ser explorada, pois a mesma não possuía estoque de crescimento. Porém, a abertura do dossel provocada pela exploração criou um ambiente favorável para o desenvolvimento da espécie, pois aos 30 anos (1979-2009) após a exploração observa-se indivíduos na classe de diâmetro $45-55 \mathrm{~cm}$, demostrando que a falta de indivíduos nas classes inferiores não é indicativo de uma provável extinção futura da epécie nesta área.

Segundo Schaaf et al. (2006), uma espécie com poucos indivíduos nas primeiras classes, mas com pequena probabilidade de morrer devido à competição, é bem provável que se mantenha na floresta. No entanto, uma espécie com grande parte dos indivíduos sujeitos a morrer, irá necessitar de um grande número de indivíduos nas classes diamétricas inferiores para se manter na comunidade. Apesar da ocorrência de ingresso na classe $45-55 \mathrm{~cm}$, no $\mathrm{Km}$ 67, o número de indivíduos na área permite concluir que a população da espécie com DAP $\geq 45 \mathrm{~cm}$ encontra-se em perigo de extinção. Assim, a população poderá desaparecer da área de manejo caso os seus dois únicos representantes morram.

H. excelsum possui característica de tolerante à sombra (LOPES et al., 2001), indicando que não há restrição quanto à sua regeneração natural em termos de radiação solar, ou seja, a regeneração é constante e compensa a mortalidade. Segundo Jardim (2015), tais espécies têm como característica uma distribuição diamétrica contínua e decrescente. Essa distribuição ocorreu no Km 114, entretanto no Km 67, a espécie teve distribuição descontínua, com poucos indivíduos nas primeiras classes de diâmetro em 1975 (Figura 3). Isso pode estar relacionado a outros fatores, como produção de sementes, dispersão, predação. Afinal se esses processos não estão sendo eficientes não haverá indivíduos para se desenvolver e chegar às classes superiores de diâmetro, tornando-se necessários estudos sobre a biologia reprodutiva desta espécie na área de estudo.

A distribuição do volume em cada classe de diâmetro no Km 114 (Figura 4) confirma o quanto o volume existente em 2014 estava distante do que havia em 1981, antes da exploração. O período de 32 anos após a exploração não foi sufuciente para a espécie recuperar seu estoque. Estatisticamente, existe diferença significativa de volume entre os dois inventários analisados, considerando um nível de significância de 5\%. Resultados semelhantes foram obtidos na mesma área da presente pesquisa por De Avila et al. (2017), que estudaram a recuperação de estoque do grupo de espécies colhidas em 1982 e concluíram que 30 anos não foram suficientes para o grupo recompor o estoque original, devido à intensidade de exploração ter sido muito alta. Portanto, nessa área, devido ao alto volume de madeira colhido, o ciclo de corte de 35 anos, previsto na legislação, não será suficiente para H. excelsum e outras espécies colhidas recuperarem seu volume. Assim, sugere-se que essas espécies não sejam colhidas no segundo corte, apesar de existir estoque disponível, para que em período mais longo o estoque das árvores em crescimento aumente, disponibilizando mais volume para futuras colheitas.
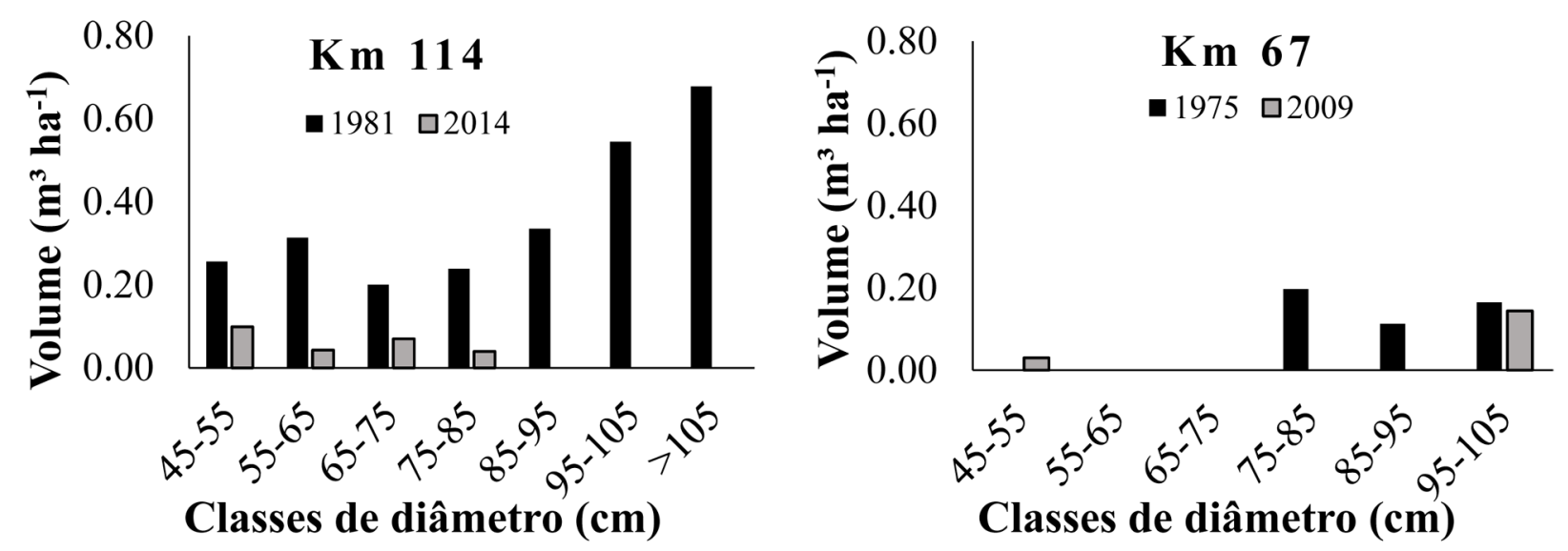

Figura 4. Volume de Hymenolobium excelsum Ducke obtido nos inventários a $100 \%$ de intensidade realizados na área experimental do Km 114 e do Km 67 na Floresta Nacional do Tapajós.

Figure 4. Volume of Hymenolobium excelsum Ducke from the $100 \%$ intensity inventories in the $\mathrm{Km} 114$ and $\mathrm{Km} 67$ experimental areas in the Tapajós National Forest. 
No Km 67 falta 0,023 $\mathrm{m}^{2}$ ha $^{-1}(37 \%)$ para a população da espécie recuperar a área basal existente antes da exploração e $0,301 \mathrm{~m}^{3} \mathrm{ha}^{-1}(36,6 \%)$ para recuperar o volume colhido na área, que segundo Costa Filho et al. (1980), foi de 0,430 $\mathrm{m}^{3} \mathrm{ha}^{-1}$. Estatisticamente, há diferença significativa entre os estoques de volume de 1975 e 2009, considerando um nível de significância de 5\%. Essa diferença é nitidamente perceptível na Figura 4, com alto estoque de volume nas classes 75-85 cm e 85-95 cm em 1975 e nenhum volume em 2009 nessas classes.

\section{CONCLUSÕES}

O período de 30 anos após a exploração florestal não foi suficiente para Hymenaea parvifolia recuperar o número de árvores, a área basal e o volume existente antes da exploração, nas duas áreas experimentais avaliadas, considerando a alta intensidade de colheita de madeira. Atualmente a espécie tem volume disponível para colheita, na área do $\mathrm{Km} \mathrm{67,} \mathrm{porém} \mathrm{não} \mathrm{possui} \mathrm{estoque} \mathrm{em}$ crescimento que possa assegurar novas colheitas em futuro próximo, necessitando de atenção especial no manejo de sua população. Na área do Km 114, a espécie possui um estoque que garante a colheita no segundo corte, porém recomenda-se que sua exploração não ocorra antes de recuperar completamente a estrutura de sua população original.

A alta intensidade de colheita não permitiu que Hymenolobium excelsum recuperasse o número de árvores existente antes da exploração nas duas áreas experimentais, no período de 30 anos. A espécie possui estoque em crescimento que garante a sua exploração nos próximos cortes, porém sugere-se que seja colhida após recuperar a estrutura de sua população original.

\section{REFERÊNCIAS BIBLIOGRÁFICAS}

BRASIL. Ministério do Meio Ambiente. Instrução Normativa n 1, de 12 de fevereiro 2015. Diário Oficial da União, Brasília, 13 fev. 2015. p.67.

BRASIL. Ministério do Meio Ambiente. Portaria no 443, de 17 de dezembro de 2014. Diário Oficial da União, Brasília, 18 dez. 2014. p. 110-121.

BRASIL. Ministério do Meio Ambiente. Instrução Normativa nº 05, de 11 de dezembro de 2006. Dispõe sobre procedimentos técnicos para elaboração, apresentação, execução e avaliação técnica de Planos de Manejo Florestal Sustentável-PMFSs nas florestas primitivas e suas formas de sucessão na Amazônia Legal, e dá outras providências. Diário Oficial da União, 12 dez. 2006. p. 155.

BRAZ, E. M., SCHNEIDER, P. R.; MATTOS, P. P.; SELLE, G. L.; THAINES, F.; RIBAS, L. A.; VUADEN, E. Taxa de corte sustentável para manejo das florestas tropicais. Ciência Florestal, Santa Maria, v. 22, n. 1, p. 137-145, 2012a.

BRAZ, E. M.; SCHNEIDER, P. R.; MATTOS, P. P.; THAINES, F.; SELLE, G. L.; OLIVEIRA, M. F.; OLIVEIRA, L. C. Manejo da estrutura diamétrica remanescente de florestas tropicais. Ciência Florestal, Santa Maria, v. 22, n. 4, p. 787-794, 2012b.

BRIENEN, R. J. W; ZUIDEMA, P. A. Incorporating persistent tree growth differences increases estimates of tropical timber yield. Frontiers in Ecology and the Environment, Washington, v. 5, n. 6, p. 302-306. 2007.

CARVAlHO, J. O. P. Subsídios para o manejo de florestas naturais na Amazônia brasileira: resultados de pesquisa da EMBRAPA/IBDF-PNPF. Documentos Embrapa. Belém, n. 43, 1987. 35 p.

CARVALHO, J. O. P. Inventário diagnóstico da regeneração natural da vegetação em área da Floresta Nacional do Tapajós. Boletim de Pesquisa, Belém, n. 2, 1980. 23 p.

CARVALHO, J. O. P.; SILVA, J. N. M.; LOPES, J. C. A.; COSTA, H. B. Manejo de florestas naturais do trópico úmido com referência especial à Floresta Nacional do Tapajós no estado do Pará. Documentos Embrapa, n. 26, 1984. 14 p.

Sci. For., Piracicaba, v. 46, n. 117, p. 41-52, mar. 2018 DOI: dx.doi.org/10.18671/scifor.v46n117.04 
Gomes et al. - Estoque de duas espécies arbóreas ameaçadas de

extinção aos 30 anos após a exploração na Amazônia oriental

COSTA, D. H. M.; SILVA, S. M. A. DA; SILVA, J. N. M. Efetividade e custo do desbaste com aplicação de arboricida em floresta natural na região do Tapajós, Pará, e Jari, Amapá. In: SILVA, J. N. M.; CARVALHO, J. O. P. DE; YARED, J. A. G. (eds.). A silvicultura na Amazônia Oriental: contribuições do projeto Embrapa/ DFID. Belém: Embrapa Amazônia Oriental/DFID, 2001. p. 339 - 352.

COSTA FILHO, P. P.; COSTA, H. B.; AGUIAR, O. R. Exploração mecanizada na Floresta Tropical Úmida sem babaçu. Belém: Embrapa-CPATU, Circular Técnica Embrapa, Belém, n. 9, 1980. 38 p.

DAUBER, E.; FREDERICKSEN, T. S; PEÑA-CLAROS, M. Sustainability of timber harvesting in Bolivian tropical forests. Forest Ecology and Management, Amsterdan, v. 214, n. 2, p. 294-304, 2005.

DE AVILA, A. L.; SCHWARTZ, G.; RUSCHEL, A. R.; LOPES, J. C.; SILVA, J. N. M.; CARVALHO, J. O. P.; DORMANN, C. F.; MAZZEI, L.; SOARES, M. H. M.; BAUHUS, J. Recruitment, growth and recovery of commercial tree species over 30 years following logging and thinning in a tropical rain forest. Forest Ecology and Management, Amsterdam, v. 385, n. 2, p. 225-235. 2017.

FERNANDES, J. M.; LOPES, C. R. A. S.; RIBEIRO, R. S.; SILVA, D. R. Leguminosae no acervo do herbário da Amazônia meridional, alta floresta, Mato Grosso. Enciclopédia Biosfera, Goiânia, v. 11, n. 21, p. 2272-2293, 2015.

FERREIRA, G. C.; HOPKINS, M. J. G.; SECCO, R. S. Contribuição ao conhecimento morfológico das espécies de leguminosae comercializadas no estado do Pará, como "angelim". Acta Amazonica, Manaus, v. 34, n. 2, p. 219 - 232. 2004a.

FERREIRA, G. C.; GOMES, J. I.; HOPKINS, M. J. G. Estudo anatômico das espécies de Leguminosae comercializadas no estado do Pará como "angelim". Acta Amazonica, Manaus, v. 34, n. 3, p. 387 - 398, 2004b.

HEINSDIJK, D.; BASTOS, A. M. Inventários florestais na Amazônia. Rio de Janeiro. Boletim do Setor de Inventários florestais, Rio de Janeiro, v. 6, p. 1-10, 1963.

HIGMAN, S.; MAYERS, J.; BASS, S.; JUDD, N.; NUSSHAUM, R. Manual do manejo florestal sustentável. Viçosa: UFV, 2015. 398 p.

IBGE - INSTITUTO BRASILEIRO DE GEOGRAFIA E ESTATÍSTICA. Manual técnico da vegetação brasileira: Sistema fitogeográfico; inventário das formações florestais e campestres; técnicas e manejo de coleções botânicas; e procedimentos para mapeamentos. Rio de Janeiro: IBGE, 2012. 275 p.

INMET - INSTITUTO NACIONAL DE METEOROLOGIA. BDMEP- Banco de Dados Meteorológicos para Ensino e Pesquisa. Disponível em: < http://www.inmet.gov.br/portal/index.php?r=bdmep/bdmep $>$ acesso em: 16 Nov. 2017.

IUCN - INTERNATIONAL UNION FOR CONSERVATION OF NATURE. IUCN Red List Categories and Criteria: Version 3.1. Cambridge: IUCN, 2012. 32 p.

JARDIM, F.C.S. Natural regeneration in tropical forests. Revista de Ciências Agrárias, Belém, v. 58, n. 1, p. 105-113. 2015.

JARDIM, F. C. S.; SOUZA, A. L.; BARROS, N. F.; SILVA, A. F.; MACHADO, C. C.; SILVA, E. Agrupamento das espécies arbóreas de uma floresta equatorial na região de Manaus-AM. Boletim FCAP, Belém, n. 26, p. 7-29, 1996.

LEÃO, N. V. M.; CARVALHO, J. O. P. Fenologia reprodutiva de 25 espécies arbóreas da Amazônia. In: SILVA, J. N. M.; CARVALHO, J. O. P. DE; YARED, J. A. G. (eds.). A silvicultura na Amazônia Oriental: contribuições do projeto Embrapa/DFID. Belém: Embrapa Amazônia Oriental/DFID, 2001. p. 117 - 128. 
LEÃO, N. V. M.; OHASHI, S.T.; FREITAS, A. D. D.; NASCIMENTO, M. R. S. M.; SHIMIZU, E. S. C.; REIS, A. R. S.; GALVÃO FILHO, A. F; SOUZA, D. Colheita de sementes e produção de mudas de espécies florestais nativas. Belém, Embrapa Amazônia Oriental, Documentos Embrapa, n. 374, 2015. 47 p.

LIMA, J. P. C.; LEÃO, J. R. A. Dinâmica de Crescimento e Distribuição Diamétrica de Fragmentos de Florestas Nativa e Plantada na Amazônia Sul Ocidental. Floresta e Ambiente, Rio de Janeiro, v. 20, n. 1, p. 70-79, 2013.

LOPES, J. C. A.; WHITMORE, T. C.; BROWN, N. D.; JENNING, S. B. Efeito da exploração florestal nas populações de mudas em uma floresta tropical úmida no município de Moju, PA. In: SILVA, J. N. M; CARVALHO, J. O. P.; YARED, J. A. G. (Eds.). A silvicultura na Amazônia Oriental: contribuições do projeto Embrapa/DFID. Belém: Embrapa Amazônia Oriental - DFID, 2001. p. 227-251.

OLIVEIRA JUNIOR, R.C.; CORREA, J.R.V. Caracterização dos solos do município de Belterra, Estado do Pará. Belém: Embrapa Amazônia Oriental, 2001. 39 p. (Documentos Embrapa, 88)

ORELlANA, E.; FIGUEIREDO FILHO, A.; PÉLlICO NETTO, S.; DIAS, A.N. Modelagem da distribuição diamétrica de espécies florestais em um fragmento de floresta ombrófila mista. Revista árvore, Viçosa, v. 38, n. 2, p. 297-308, 2014.

PARIONA, W.; FREDERICKSEN, T.S.; LICONA, J.C. Natural regeneration and liberation of timber species in logging gaps in two Bolivian tropical forests. Forest Ecology and Management, Amsterdan, v. 181, n. 3, p. 313-322. 2003.

PUTZ, F.E.; ZUIDEMA, P.A.; SYNNOTT; T.; PENÃ-CLAROS, M.; PINARD, M.A.; SHEIL, D.; VANCLAY, J.K.; SIST, P.; GOURLET-FLEURY, S.; GRISCOM, B.; PALMER, J.; ZAGT, R. Sustaining conservation values in selectively logged tropical forests: the attained and the attainable. Conservation Letters, v. 5, n. 4, p. 296-303, 2012.

REIS, L. P.; RUSCHEL, A. R.; COELHO, A. A.; LUZ, A. S.; SILVA, R. C. V. M. Avaliação do potencial madeireiro na Floresta Nacional do Tapajós, após 28 anos da exploração. Pesquisa Florestal, Colombo, v. 30, n. 64, p. 265-281, 2010.

SALOMÃO, R. P.; ROSA, N. A.; MORAIS, K. A. C. Dinâmica da regeneração natural de árvores em áreas mineradas na Amazônia. Boletim Museu Paraense Emílio Goeldi, Belém, v. 2, n. 2, p. 85-139, 2007.

SALOMÃO, R. P.; ROSA, N. A.; NEPSTAD, D. C.; BAKK, A. Estrutura diamétrica e breve caracterização ecologíca econômica de 108 espécies arbóreas da floresta amazônica brasileira. Interciencia, Caracas, v.20, n.1, p. 2029. 1995.

SCHAAF, L. B.; FIGUEIREDO FILHO, A.; GALVÃO, F.; SANQUETTA, C. R. Alteração na estrutura diamétrica de uma floresta ombrófila mista no período entre 1979 e 2000. Revista Árvore, Viçosa, v. 30, n. 2, p. 283-295, 2006.

SCHWARTZ, G.; PEÑA-CLAROS, M.; LOPES, J. C. A.; MOHREN, G. M. J.; KANASHIRO, M. Mid-term effects of reduced-impact logging on the regeneration of seven tree commercial species in the Eastern Amazon. Forest Ecology and Management, Amsterdan, v. 274, n. 1, p. 116-125, 2012.

SILVA, B. M. S.; CESARINO, F. Germinação de sementes e emergência de plântulas de jutaí (Hymenaea parvifolia Huber). Revista Brasileira de Plantas Medicinais, Campinas, v. 18, n. 1, supl. I, p. 256-263, 2016.

SILVA, B. M. S.; LIMA, J. D.; DANTAS, V. A. V.; MORAES, W. S.; SABONARO, D. Z. Efeito da luz no crescimento de mudas de Hymenaea parvifolia Huber. Revista Árvore, Viçosa, v. 31, n. 6, p. 1019-1026, 2007.

SILVA, J. N. M.; CARVALHO, J. O. P.; LOPES, J. C. Inventário florestal de uma área experimental na Floresta Nacional do Tapajós. Boletim de Pesquisa Florestal, Curitiba, n. 10/11, p. 38-110, 1985. 
Gomes et al. - Estoque de duas espécies arbóreas ameaçadas de

extinção aos 30 anos após a exploração na Amazônia oriental

SILVA, J. N. M.; CARVALHO, J. O. P.; LOPES, J. C. A.; CARVALHO, M. S. P. Equações de volume para a Floresta Nacional do Tapajós. Boletim de Pesquisa Florestal, Colombo, n. 8/9, p. 50-63, 1984.

VIEIRA, A. H.; MARTINS, E. P.; PEQUENO, P. L. L.; LOCATELLI, M. Interpretação Fenológica de uma Floresta Ombrófila Aberta em Porto Velho, RO. Boletim de pesquisa e desenvolvimento, Porto Velho, v. 12, 2002.13 p.

Recebido em 09/04/2017

Aceito em 09/10/2017 